\title{
Gene Editing, Sexual Reproduction, and the Arts: The Present, the Future, and the Imagined
}

\author{
Roberta Buiani
}

In recent years, popular culture has been graced with countless news announcing novel developments in genome editing. While many experiments are still in their early stages, genome editing seems very promising. Often betraying a sensationalist and triumphant tone, news coverage focuses on the potentials that these developments will have for the advancement of the human species, i.e., the eradication of disease, the extension of life, the improvement of the body and its appearance, etc. The future looks hopeful and unproblematic according to these accounts. On the opposite end of the spectrum, some may wonder whether these developments pose a potential worsening of the human condition: Are these developments safe? What are the ethical implications? Who will benefit from these developments? Given today's social divisions and cultural conflicts, these voices predict a rather unpromising future and warn against the pursue of innovation at any cost.

What these radically opposed positions share is that they all tend to make predictions and look at the future: a promising, improved future on the one hand, and a discouraging, problematic future on the other hand.

We propose before we look at what genome editing will bring us in the future to look how it affects the present. In particular, we ask: What are the unresolved issues in the present that we should address before launching ourselves into the future? Can we find interesting ways to draw attention to them and redress how they are currently treated through creative interventions? More specifically, We would like to explore issues regarding sexual reproduction, fertility, and sexual technologies. Artistic interventions pertaining to these topics, in addition to raising awareness about sexism, sexual rigidity, and the medicalization of the body, may also be suggestive of ways in which we might rethink the role of human enhancement and genome editing in sciences as well as in everyday life. We believe that present issues in gynecology, hormonal management, human enhancement, and sexual and cultural identity may be addressed, redressed, hacked, and reimagined through the arts.

\author{
R. Buiani $(\triangle)$ \\ ArtSci Salon, Fields Institute, Toronto, ON, Canada
}

